\title{
Analysis of Charge Relaxation in the Modified Films of the polyimide
}

\author{
Margarita E. Borisova ${ }^{*}$, Almaz Kamalov ${ }^{2}$ \\ ${ }^{1,2}$ Institute of Energy and Transport Systems, Peter the Great St. Petersburg Polytechnic University, \\ Russian Federation
}

\begin{abstract}
The aim of this work is the experimental and theoretical study of charge relaxation processes in the films of polyimide coated on both sides with lacquer PTFE (PMF-2), charged in a corona discharge at negative polarity in ambient conditions. The theoretical analysis is based on the model of quasi-continuous distribution of traps with respect to their energies. This paper shows that the stability of the electret state is caused by deep traps that are localized in a Teflon coating.
\end{abstract}

Keywords - Charge relaxation, charge stability, depolarization currents, electret, energy of activation, homo-charge, polyimide films, polymer surface modification, traps.

\section{INTRODUCTION}

Composite polymer corona-electrets occupy a special place among active dielectrics in modern electrical engineering. The combination of increased thermostability [1], mechanical, electrical and optical properties, possessed by polyimide, can increase the lifetime of the electret.

However, up to now polyimide was not used as the electret. The usage of polyimide films (PM) in electronic devices is limited by their low water resistance [2]. This work is devoted to the study of accumulation and charge relaxation in the composite film PMF-2 (polyimide coated on both sides with lacquer PTFE), which can be used as an active dielectric.

The relaxation of the electric charge in the films of the PMF2 was studied by thermally stimulated depolarization (TSD). TSD spectra provide information about the mechanism of accumulation and relaxation of charge. It requires an analysis based on different physical models for getting information from the TSD curves.

The method of charging the polymer films in the corona discharge becomes the most common in practice. This method is characterized by simplicity and accessibility, the possibility of obtaining electrets with a given value of the surface charge density.

\section{EXPERIMENT}

\section{Polyimide-fluoroplastic film}

A polyimide film (PM) is coated with PTFE resin and sintered at a temperature above $300^{\circ} \mathrm{C}$ on one or two sides. To improve the adhesion properties of the polyimide film to the PTFE varnish, it is pre-processed in a corona discharge. This film combines the excellent properties of polyimide film and
FEP resin: heat resistance, cold resistance, radiation resistance, good electrical properties. PMF-2 is successfully used in a wide temperature range: from $-80{ }^{\circ} \mathrm{C}$ to $+260{ }^{\circ} \mathrm{C}$.

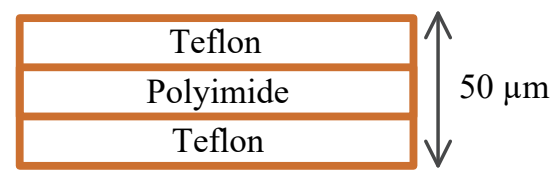

Fig. 1. PMF-2 film structure

The high thermal and chemical stability of PI is due to two reasons:

1) the high resonance energy of the benzene rings (Fig. 2) due to delocalization of the $\pi$-electrons and the great number of resonance structures;

2) strength of the imide bonds, resulting from the competitive $n-\pi$ conjugation between the carbonyl group and the non-pair electron couple from the nitrogen atom as well as from the conformation state of the 5-member imide ring. The lack of Baer's angular torsion is due to the fact that all $\mathrm{C}$ and $\mathrm{N}$ atoms are in a sp2 hybrid state with valent angle of $120^{\circ}$ and planar conformation of the ring.

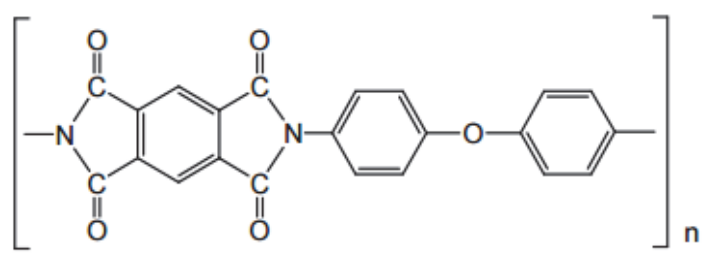

Fig. 2. Chemical formula of a molecule of polyimide.

\section{Electret fabrication}

Dielectric charging is made in a corona discharge under ambient conditions, with negative polarity of corona electrode. Setup for sample charging is shown in Fig. 3.

\footnotetext{
${ }^{*}$ Corresponding author.

E-mail address: vladimirl.borisov@gmail.com
} 


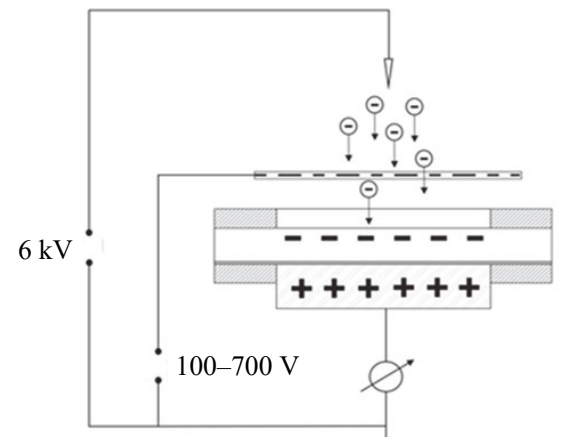

Fig. 3. Setup for sample charging.

It is known [3] that an exchange of charges between the ions that come from the gas discharge and a polymer film surface according to the mechanism of Auger neutralization occurs in the process of charging. As a result, the electret homo-charge appears. The localization of the charge carriers occurs in the traps in a thin surface layer of the dielectric.

To assess the stability of the electret state of the coronaelectret, we studied thermally stimulated depolarization currents of films PMF-2 and PM. The structure of the film PMF-2 is shown in Fig. 1.

PM and PMF-2 films are charged to $400 \mathrm{~V}$ with a negative corona needle for 30 seconds under ambient conditions. The TSD currents were measured at linearly rising temperature with the rate $\mathrm{d} T / \mathrm{d} t=2 \mathrm{~K} / \mathrm{min}$ and in open circuit when PTFE films (thickness $40 \mu \mathrm{m}$ ) were employed. In accordance with GOST 25209-82, automated setup for electret thermal analysis comprises the following parts, shown in a block diagram form in Fig. 4.

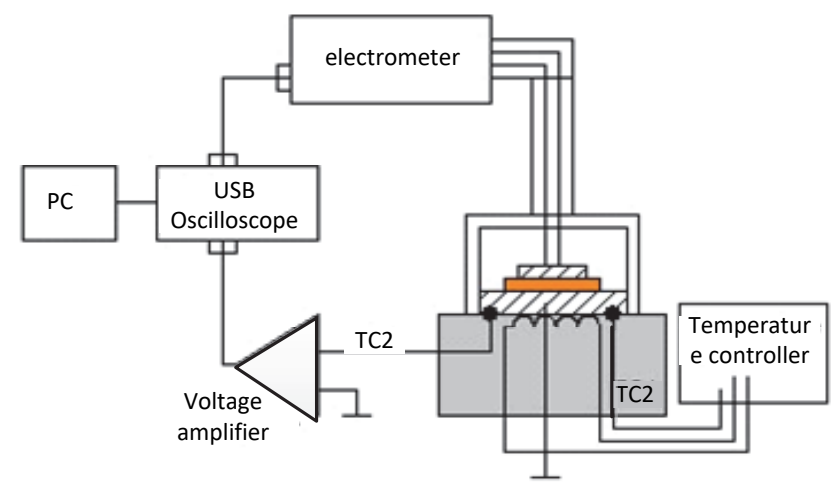

Fig. 4. Experimental setup for TSD measurement.

For small currents measuring in the automated setup, we use electrometric voltmeter-electrometer with a sensitivity of $10 \mathrm{~A}$ to $14 \mathrm{~A}$. The electret was heated by thermal contact with the lower electrode-heater, in which the spiral of nichrome wire was mounted. The temperature was maintained and controlled by an automatic programmer Termodat-14E2. Current and thermo-emf signals pass into the USB oscilloscope, which converts and outputs the signals to the computer, which shows the graphic - current versus temperature.

Experimentally measured TSD spectra are shown in Fig. 5.

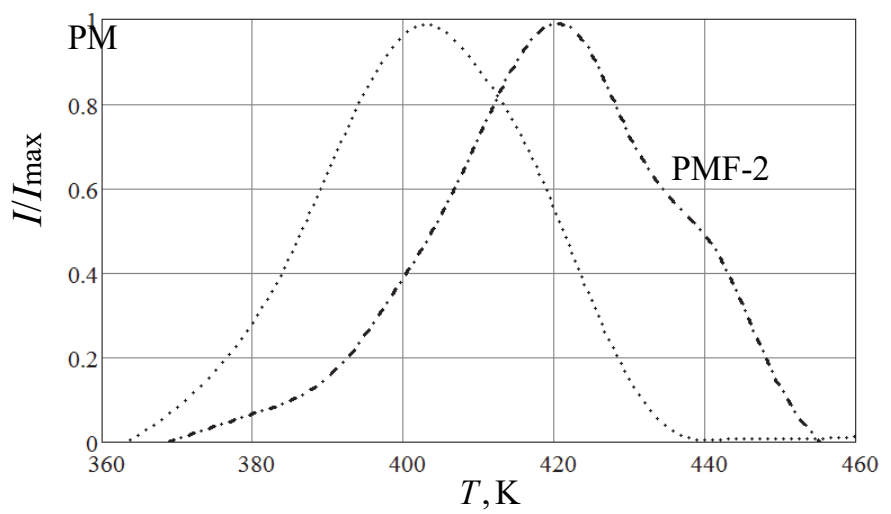

Fig. 5. Spectra of TSD currents of films PM and PMF-2.

It is seen that the structure of PMF-2 film has higher thermal stability compared to the PM electret film.

The TSD currents in PMF-2 were measured at linearly rising temperature with the rate $\mathrm{d} T / \mathrm{d} t=2 \mathrm{~K} / \mathrm{min}$ and $\mathrm{d} T / \mathrm{d} t=6 \mathrm{~K} / \mathrm{min}$ in open circuit when PTFE films (thickness $40 \mu \mathrm{m}$ ) were employed.

Measuring at different heating rates allows using the Simmons theory to calculate the energy spectrum of traps charge carriers. The depth of traps standing determines the stability of the electret dielectric state.

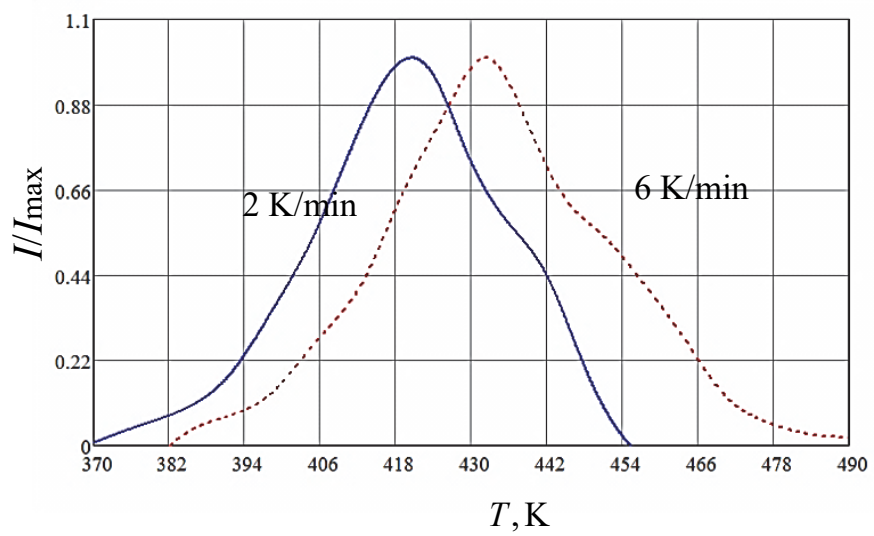

Fig. 6. Experimental dependence of TSD currents (PMF-2 films) at different heating velocities.

Fig. 6 shows the complexity of the TSD spectra and the tendency of shifting to higher temperatures with the increasing of heating rate.

It is known [4] that in the non-polar fluorinated polymers, the relaxation of homo-charge is entirely caused by the release of the carrier from the surface traps and its drift through the thickness of the dielectric. Therefore, it can be assumed that the majority of traps are located in the Teflon coating. The presence of traps is usually associated with specific surface defects: circuit breaking, adsorbed molecules, and oxidation products [5]. 


\section{ANALYSIS OF THE TSD SPECTRA}

TSD spectra have complex patterns with a broad maximum. Therefore, it is reasonable to analyze the current TSD on the base of the model of quasi-continuous energy traps distribution. We use the Simmons theory to calculate the function of trap energy distribution[6].

For the processing of the experimental data, we use computer software (Mathcad 15.0), allowing to automate the process of calculation and improve the measurement accuracy of the parameters of relaxation processes that determine the electret effect.

As a result of corona-charging, homo-charge accumulates in the traps (with $n_{\mathrm{s} 0}$ concentration) localized in the surface layer of Teflon coating structure PMF-2.

The current density at the thermally stimulated depolarization mode with a linear heating rate $\beta$ is described by the formula:

$$
\begin{aligned}
& j=q n_{\mathrm{s} 0} \int_{W_{\mathrm{a} 0}}^{W_{\mathrm{a}}} f_{0}^{*}\left(W_{\mathrm{a}}\right) N^{*}\left(W_{\mathrm{a}}\right) \omega_{\mathrm{s}} \exp \left(-W_{\mathrm{a}} / k T\right) \\
& \cdot \exp \left[-\frac{1}{\beta} \int_{T_{0}}^{T} \omega_{\mathrm{s}} \exp \left(-W_{\mathrm{a}} / k T\right) \mathrm{d} T\right] \mathrm{d} W_{\mathrm{a}},
\end{aligned}
$$

where $f_{0}\left(W_{\mathrm{a}}\right)$ - parameter of the initial traps distribution; $N\left(W_{\mathrm{a}}\right)$ - the function of the quasi-continuous distribution of the surface traps with respect to the activation energies $W_{\mathrm{a}}$.

The product of $f_{0}\left(W_{\mathrm{a}}\right) N\left(W_{\mathrm{a}}\right)$ is a function of the distribution of occupied surface traps with respect to activation energies. According to this model, the relaxation of the electret is determined solely by the process of activation of homocharge from traps. For further analysis, the authors [7] introduced in consideration the distribution function, normalized to the total concentration $n_{\mathrm{s} 0}$ :

$$
f_{0}^{*}\left(W_{\mathrm{a}}\right) N^{*}\left(W_{\mathrm{a}}\right)=\left(n_{\mathrm{s} 0}\right)^{-1} f_{0}\left(W_{\mathrm{a}}\right) N\left(W_{\mathrm{a}}\right) .
$$

Simmons showed [7] that the integrand

$$
\begin{aligned}
& P\left(W_{\mathrm{a}}, T\right)=\omega_{\mathrm{s}} \exp \left(-W_{\mathrm{a}} / k T\right) \\
& \cdot \exp \left[-\frac{1}{\beta} \int_{T_{0}}^{T} \omega_{\mathrm{s}} \exp \left(-W_{\mathrm{a}} / k T\right) \mathrm{d} T\right]
\end{aligned}
$$

has a sharp maximum at energy $W_{\mathrm{a}}^{\mathrm{m}}$, equal to

$$
W_{\mathrm{a}}^{\mathrm{m}}=T\left(\lg \frac{\omega_{\mathrm{s}}}{\beta} b+a\right)-c .
$$

Also, Simmons showed that the result of the numerical solution of the integral equation leads to the values of numerical coefficients $a=3.2 \cdot 10^{-4}, b=1.92 \cdot 10^{-4}, c=0.015$.

Furthermore, it was shown that $P\left(W_{\mathrm{a}}, T\right)$ can be approximated by the expression

$$
P\left(W_{\mathrm{a}}, T\right) \approx D \delta\left(W_{\mathrm{a}}-W_{\mathrm{a}}^{\mathrm{m}}\right),
$$

where $D-$ is the area of the delta function, weakly dependent on the temperature:

$$
D=\frac{1.2 \beta W_{\mathrm{a}}^{\mathrm{m}}}{T} .
$$

As a result, the TSD current is determined by the equation

$$
\begin{aligned}
& j(T)=q n_{\mathrm{s} 0} D f_{0}^{*}\left(W_{\mathrm{a}}\right) N^{*}\left(W_{\mathrm{a}}\right) \Rightarrow \\
& \Rightarrow f_{0}\left(W_{\mathrm{a}}\right) N\left(W_{\mathrm{a}}\right)=j(T) / q D .
\end{aligned}
$$

Using equations (1.1) and (1.2), we calculated the energy distribution function of the traps shown in Fig. 7.

The frequency factor was determined from the experimental curves (Fig. 1) by the shift of the TSD maximum:

$$
\lg \omega_{\mathrm{s}}=\frac{T_{2}^{*} \lg \beta_{2}-T_{1}^{*} \lg \beta_{1}}{T_{2}^{*}-T_{1}^{*}}-a \Rightarrow \omega_{\mathrm{s}}=1.09 \cdot 10^{14} c^{-1},
$$

where $T_{1}^{*}$ and $T_{2}^{*}$ - the temperatures of TSD current maxima, measured at the heating rates $\beta_{1}$ and $\beta_{2}$, respectively.

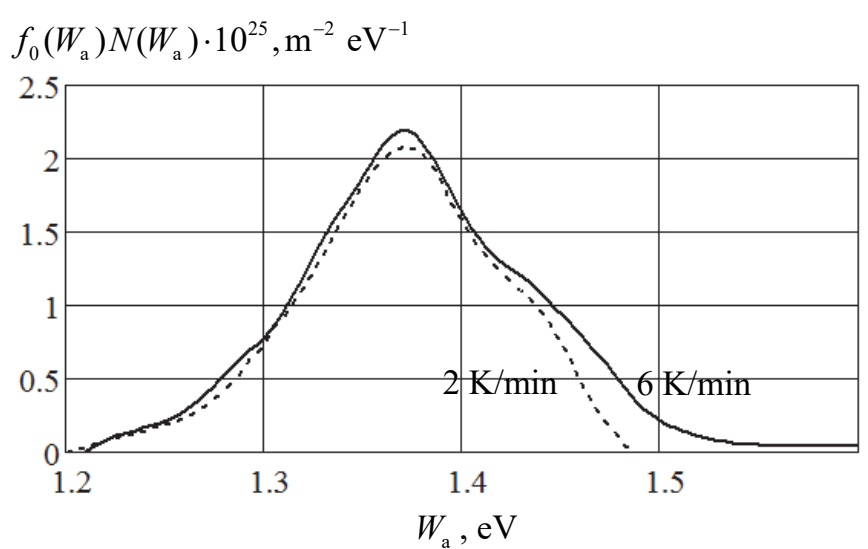

Fig. 7. The function of the distribution of the activation energies traps, calculated from the measured experimental curves.

The area of traps activation energy (Fig. 6) is in the range from $1.2 \mathrm{eV}$ to $1.55 \mathrm{eV}$, and the activation energy corresponding to the maxima of distribution is $1.37 \mathrm{eV}$. The distribution function shows the concentration of the filled traps per unit area of the dielectric.

\section{CONCLUSION}

This paper shows that the charge is accumulating in deep-energy traps in the PTFE layer. Activation energy traps are in the range from $1.55 \mathrm{eV}$ to $1.2 \mathrm{eV}$, and the frequency factor is equal to $\omega_{\mathrm{s}}=1.09 \cdot 10^{14} c^{-1}$. We have previously shown [8] that the PTFE coating in the structure of PMF-2 protects the polyimide film from moisture, which greatly increases the thermal stability of the accumulated charge.

This result explains the stability of the electret state in a layered structure at the molecular level and allows us to control the charge relaxation and, therefore, homo-charge stability in corona-electrets. 
The multilayer structure of TFM-2 can be used for the production of the corona-electrets, which will improved thermal stability under high humidity conditions.

\section{REFERENCES}

[1] M. I. Bessonov, M. M. Koton, V. V. Kudryavtsev, and L. A. Laius, Polyimides: Thermally stable polymers, Consultants Bureau, New York, 1987, p. 329.

[2] G. M. Sessler, Ed., "Electrets," in Topics in Applied Physics, 1980. https://doi.org/10.1007/978-3-540-34846-7

[3] A. Georgiev, E. Spassova, J. Assa, and G. Danev, "Preparation of Polyimide Thin Films by Vapour Deposition and Solid State Reactions," in Polymer Thin Films, Apr. 2010. pp. 71-92.

https://doi.org/10.5772/8389

[4] G. M. Sessler, C. Alquié, and J. Lewiner, "Charge distribution in Teflon FEP (fluoroethylenepropylene) negatively corona-charged to high potentials," Journal of Applied Physics, vol. 71, no. 5, pp. 2280-2284, Mar. 1992. https://doi.org/10.1063/1.351127

[5] A. A. Rychkov and V. G. Boitsov, "Charge relaxation in PTFE-Al structures having interfacial region modified by the glow discharge," 10th International Symposium on Electrets (ISE 10). Proceedings (Cat. No. 99 CH36256). Athens, 1999, pp. 91-94. https://doi.org/10.1109/ise.1999.831952

[6] J. G. Simmons, G. W. Taylor, and M. C. Tam, "Thermally Stimulated Currents in Semiconductors and Insulators Having Arbitrary Trap Distributions," Physical Review B, vol. 7, no. 8, pp. 3714-3719, Apr. 1973. https://doi.org/10.1103/physrevb.7.3714

[7] J. G. Simmons and M. C. Tam, "Theory of Isothermal Currents and the Direct Determination of Trap Parameters in Semiconductors and Insulators Containing Arbitrary Trap Distributions," Physical Review B, vol. 7, no. 8, pp. 3706-3713, Apr. 1973.

https://doi.org/10.1103/physrevb.7.3706

[8] A. M. Kamalov and M. E. Borisova, "The influence of moisture on charge relaxation in modified polyimide films," St. Petersburg State Polytechnical University Journal. Physics and Mathematics, vol. 248, no. 3, pp. 33 38, Sep. 2016. https://doi.org/10.5862/jpm.248.4 